\section{Interpreting theories}

To interpret a formal system is to give it a reading by assigning some values to the formulas (and in more advanced cases to other things, such as names and predicates). This will then produce an account of the meaning of the logical symbols ( $\&$ and $\rightarrow$ in the example) in terms of their effects on the values of formulas and so on. Every interpretation picks out some set of formulas as being "true." If every formula in a set $S$ is true for a given interpretation then that interpretation is said to be a model of S. A theory, in the sense provided by a formal logic, is a set of formulas in the appropriate language such that whenever any argument from $A_{1} \ldots A_{n}$ to $B$ is valid in the logic, and all of $A_{1} \ldots A_{n}$ are in the theory, so is $B$. The items in a theory are called its theorems. A model of a theory is therefore an interpretation for which everything said by that theory is true.

For example, we can interpret our calculus of $\&$ and $\rightarrow$ in terms of the concept of "information." Suppose that there are some "pieces of information" and that we can pick out various sets of these as possible "states of information." It does not matter formally what these are: only the structure of the idea counts. One possible state of information - call it $T$ (for True)-is supposed to be the information actually given by the real world. Now each atom $(P, Q$, and so on) is interpreted as conveying a piece of information. For a given interpretation in this sense each formula is either "warranted" or "not warranted" by each state of information as follows:

(1) An atom is warranted by state $S$ if and only if its information is in $\mathrm{S}$.

(2) A conjunction A \& B is warranted by $S$ if and only if both of $A$ and $B$ are warranted by $S$.
(3) A conditional $A \rightarrow B$ is warranted by $S$ if and only if $B$ is warranted by every state that includes $S$ and warrants $A$.

A formula is true for an interpretation if and only if it is warranted by $T$ according to that interpretation. An argument form is valid provided that its conclusion is true for every interpretation for which its premises are true. It can be shown that validity thus defined coincides with derivability according to the five rules given above in the sense that we get the same set of valid argument forms whether we define the logic as a system of derivations or as a theory of information. This fact is a completeness theorem for the system in question. This system differs slightly from the one more usually found in introductory texts, as will be noted below in section IV.

What has been set out in this section is, of course, a very small part of formal logic. It can be elaborated to take account of much more complex reasonings, including arguments of the kind given in section I and many others. Logical theory since 1900 has been partly a matter of formulating such elaborations and partly concerned with the investigation of concepts arising - the theory of sets, model theory, proof theory, parts of abstract algebra, recursion theory, and so on. Next week we will leave aside the technicalities of mathematical logic and return to considering the ways in which logic has to do with medicine.

\section{References}

1 Kneale W, Kneale M. The development of logic. Oxford: Oxford University Press, 1962:232. 2 Fitch FB. Symbolic logic; an introduction. New York: Ronald Press, 1952.

3 Kalish D, Montague R. Logic: techniques of formal reasoning. New York: Harcourt, Brace and World, 1964.

Contemporary Themes

\title{
Waiting list statistics. II: an estimate of inflation of waiting list length
}

\section{A LEE, B DON, M J GOLDACRE}

\begin{abstract}
The discrepancy between the length of the waiting list and eventual admissions from the waiting list was investigated by comparing data from two different sources of routine statistics in the Oxford region. It was estimated that about $28 \%$ of the waiting list comprised patients who were not eventually admitted to hospital within the region.
\end{abstract}

\section{Methods}

We studied figures for general surgery, trauma and orthopaedic surgery, ear, nose, and throat surgery, gynaecology, ophthalmology, and plastic surgery from 1974 to 1983 in the Oxford region. These specialties accounted for about nine tenths of the patients on the waiting list. Data were included for the five districts in the region that collected data for the Hospital Activity Analysis, including reasonably complete data on waiting times, during the whole study.

\begin{abstract}
Introduction
Several studies have indicated that routine returns on the number of patients on waiting lists overestimate the numbers of patients who will eventually be admitted to hospital. ${ }^{1-4}$ The Department of Health and Social Security has asked health authorities to review and validate their inpatient waiting lists and has suggested that at least one tenth of all patients on waiting lists will not eventually require admission. ${ }^{5}$ We investigated the discrepancy between the length of the waiting list and the number of eventual admissions from the waiting list by comparing the data from two different sources of routine statistics.
\end{abstract}

\author{
Unit of Clinica \\ M J GOLDACRE, BM, FFCM, director \\ Oxford Regional Health Authority, Headington, Oxford OX3 7LF \\ B DON, BSC, DPHIL, regional statistician \\ Correspondence to: Dr Goldacre.
}


The numbers of patients on the waiting list at the end of each quarter are recorded routinely in the SBH 203 returns (31 March and 30 September), the SH3 returns (31 December), and mid-year hospital statistical returns ( 30 June). These counts provide a census of the total number of patients recorded at the time as being on the waiting list, including those who have a booked appointment. We used these as our first source of data and will refer to them as the waiting list returns. From September 1979 onwards, as instructed by the DHSS, these returns excluded day cases.

For our second source of data we used the Hospital Activity Analysis to obtain counts of patients who have been admitted to hospital. The Hospital Activity Analysis provides data on all inpatients admitted and also includes the source of the admission, which enabled us to identify admissions from the waiting list and booked admissions. The Hospital Activity Analysis includes the date of admission and, for patients admitted from the waiting list, the date on which such patients were first placed on the waiting list. Unfortunately, it does not record the date on which patients who have a booked admission were first placed on the list. These cases have therefore been excluded from our analysis. Only about one tenth of all elective admissions (that is, booked and from the waiting list), however, were classed as "booked" in the five districts and six specialties analysed. Day cases were excluded from our analysis after June 1979 to ensure compatibility with SBH 203 definitions.

From data from the Hospital Activity Analysis it is possible to reconstruct a census of the number of patients eventually admitted who would have been on the waiting list at any given point in the past. We will refer to these figures as the Hospital Activity Analysis waiting list figures. At each quarterly reference date the difference between the waiting list returns and the Hospital Activity Analysis waiting list figures can be used to estimate the proportion of patients on the waiting list who were not subsequently admitted within the region (within a given time). We analysed the waiting list returns and the Hospital Activity Analysis waiting list figures from 1974 to 1983 using the Hospital Activity Analysis to include admissions up to 31 December 1986.

\section{Results}

The figure shows the total number of patients in each quarter who were recorded in the waiting list returns. It also shows the Hospital Activity Analysis reconstruction of the numbers of patients on the waiting list at the end of each corresponding quarter who were destined to be admitted subsequently as inpatients before the end of 1986. The Hospital Activity Analysis waiting list figures were clearly considerably lower than the waiting list returns throughout the period. Though the waiting list returns and the Hospital Activity Analysis waiting list figures were derived from independent sources, the trends in the two ran remarkably parallel. There was a significant positive correlation between quarterly changes in the waiting list returns and changes in the Hospital Activity Analysis waiting list $(r=0 \cdot 78$, $\mathrm{df}=37, \mathrm{p}<0.001$ ).

In the early quarters of the study-that is, March 1974 to June 1975-the waiting list returns were about $40 \%$ higher than the Hospital Activity Analysis waiting list figures. There was a sharp decline in this percentage between June and September 1975 and a gradual increase again thereafter.

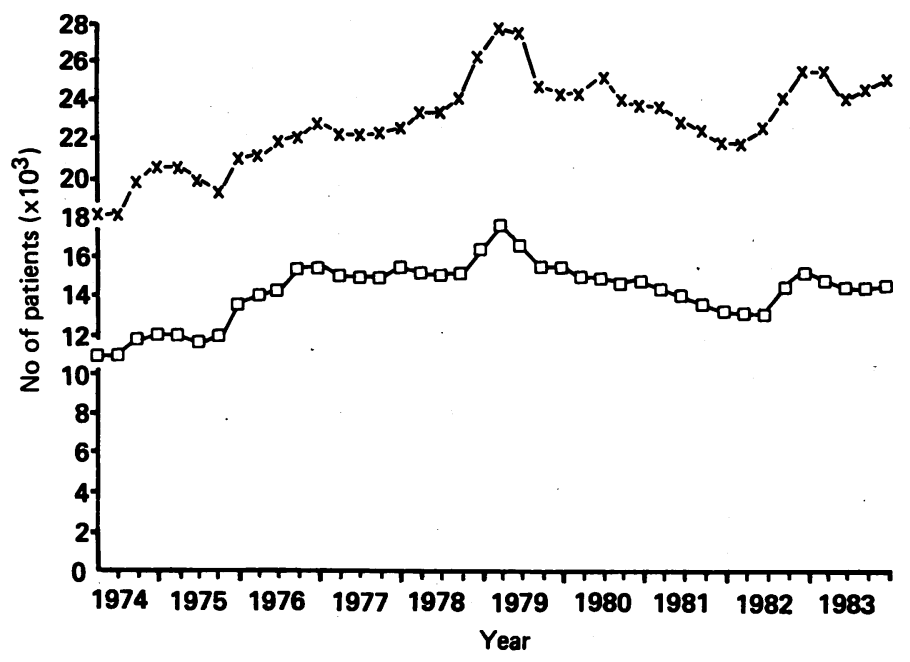

Number of patients on waiting list at end of each quarter as measured by SBH 203 returns $(X)$ and by reconstructions from Hospital Activity Analysis data ( $\square$ ).
The average difference between the two sets of data from 1974 to 1983 was $37 \cdot 8 \%$. Within this difference, however, an adjustment needs to be made to take account of missing data on waiting times. About one tenth of elective admissions were booked patients who were excluded from the Hospital Activity Analysis waiting list data because their waiting times were unavailable but who could not be separated from the waiting list returns. In addition, about $3 \%$ of all patients admitted from the waiting list in the Hospital Activity Analysis did not have a recorded date of placement on the waiting list or date of admission. These cases have been excluded from the Hospital Activity Analysis figures but they will have been included in the returns. Correcting to take account of these the estimated likely discrepancy between the sets of figures, averaged over the period, was approximately $28 \%$.

\section{Discussion}

This approach is a fairly simple method of estimating in retrospect the number of people on a waiting list who were not admitted to hospitals covered by the waiting list during a defined period. In some respects it is an extension of the method reported by Williams et al. ${ }^{6}$ There are, however, two difficulties in comparing the waiting list returns and the data from the Hospital Activity Analysis.

Firstly, it is difficult to take account of some differences in definition between the two sets of data concerning the classification of "waiting list" patients. In addition to the definitions of booked cases and day cases already discussed there are some minor differences between the sets of data in the way that, for example, "deferred admissions" are classified.' As Williams et al point out, the influence of such anomalies is difficult to judge without detailed local investigation at the relevant times. ${ }^{6}$ It is clear, however, that a sizable fraction of the waiting list during the study comprised patients who were not subsequently admitted.

Secondly, there was a decline between September 1975 and March 1976 in the proportion of people on the waiting list who were not subsequently admitted to hospital. In 1975 a DHSS circular advised health authorities to review their waiting lists and to report back on the exercise in September 1976. ${ }^{7}$ The decline at this time no doubt reflects the removal from the waiting list of the names of patients who were considered no longer to require admission.

The patients who were not eventually admitted would make up several different categories. They would include patients whose condition had improved sufficiently so that admission for treatment was no longer necessary; those who had been treated outside the region; those who had eventually been treated privately; those who had simultaneously been on more than one waiting list in the region for the same condition; those who had migrated from the region; those whose condition had deteriorated sufficiently so that they were admitted as emergency cases; and those who had died while awaiting admission to hospital. As the data from the Hospital Activity Analysis covered a fixed, limited period of time there would also of course have been patients who were not admitted but who remained on the waiting list and still required admission. More detailed studies than ours would be necessary to estimate the proportion of patients in each of these categories.

We cannot judge whether the districts studied are typical of the country as a whole in respect of data on waiting lists. Other health authorities may wish to use similar methods to those described in this paper to estimate the likely extent of inflation of local waiting lists.

\section{References}

1 Lourie JA. Notes on orthopaedic waiting list. Br $\mathcal{C}$ Clin Pract 1978;32:224-5.

2 Cocking J. In-patient waiting lists. Health and Social Services foumal 1974;85:1102-3.

3 Donaldson LJ, Maratos JI, Richardson RA. Review of an orthopaedic in-patient waiting list. Health Trends 1984;16:14-5.

4 Dalton KJ. Surgical waiting lists. Br Med $\mathcal{Y}$ 1984;289:495.

5 Department of Health and Social Security. Validation of in-patient waiting lists. London: DHSS 1984. (DA(84)30.)

6 Williams DRR, West RJ, Hagard S, Dias AFS. Waiting list monitoring using information from Hospital Activity Analysis and SBH 203 returns. Comsunurity Medicine 1983;5:311-6.

7 Department of Health and Social Security. Reduction of waiting times for in-patients admission: management arrangements. London: DHSS, 1975. (HSC(1S)181.)

(Accepted 11 August 1987) 